\title{
Measurement of Brain Structures Based on Statistical and Geometrical 3D Segmentation
}

\author{
Miguel Ángel González Ballester, Andrew Zisserman, and Michael Brady \\ Robotics Research Group, Dept. of Engineering Science \\ University of Oxford, Oxford OX1 3PJ, UK \\ $\{$ magb,az,jmb\}@robots.ox.ac.uk
}

\begin{abstract}
In this paper we present a novel method for three-dimensional segmentation and measurement of volumetric data based on the combination of statistical and geometrical information. We represent the shape of complex three-dimensional structures, such as the cortex by combining a discrete $3 \mathrm{D}$ simplex mesh with the construction of a smooth surface using triangular Gregory-Bézier patches. A Gaussian model for the tissues present in the image is adopted, and a classification procedure which also estimates and corrects for the bias field present in the MRI is used. Confidence bounds are produced for all the measurements, thus obtaining bounds on the position of the surface segmenting the image. Performance is illustrated on multiple sclerosis phantom data and on real data
\end{abstract}

\section{Introduction}

The segmentation of three-dimensional structures is fundamental to medical vision applications. Voxel-based [16], slice-based $[1,12,14]$, and deformable 3D surface methods $[6,15,18]$ have been proposed, the latter being preferred because of the intrinsically three-dimensional nature of the data sets and the flexibility of having a surface segmenting the objects in it. The shapes of some structures to be segmented, such as the brain cortex, poses an additional problem, since the shape representation must be able to cope with such complexity.

Often, and central to this article, in clinical studies the size of the effect to be studied is small relative to the voxel size. This is the case, for example, when analysing the time evolution of multiple sclerosis lesions during drug treatment or patient therapy [4], and also when assessing differences in brain asymmetry associated with schizophrenia [5,7]. Accuracy becomes critical, necessitating confidence bounds for every measurement obtained from the data sets. To provide clinically meaningful results, the width of the confidence interval should be significantly smaller than the size of the anatomical effect to be studied. To this end, we describe a method for 3D segmentation from volumetric data combining both a statistical model of the tissues present in the image and a geometric template of the expected shape, and we explain how volume measurements, together 
with confidence intervals, are obtained from it. Following [11] and [20], the voxel intensities corresponding to a certain tissue type are modelled as a Gaussian distribution with small variance. Two main problems arise when using this model to classify voxels into tissue types, namely corrupting bias fields, and partial volume effects. Both are addressed in our method, which estimates and corrects for the bias field and regards partial volumes as an indicator of boundary location.

Complex biological shapes require a shape modeling technique that is powerful enough to capture sufficient detail, flexible enough to be adapted easily to segment the data, and able to construct higher-level shape descriptors. We use the simplex mesh [6] in an initial step, followed by the construction of a smooth surface using triangular Gregory-Bézier patches [17]. Segmentation of the data is guided by the probabilities computed in the statistical classification, a set of forces implemented on the simplex mesh, and a template of the expected shape built by applying a principal component analysis (PCA) to a set of pre-segmented structures.

The following section describes the statistical model and the bias correction algorithm used to classify the voxels and to detect partial volume effects. Next, the shape representation model, fitting process, and error bound computation are explained. Finally, a set of results are shown for phantom and real data.

\section{Statistical model}

A Gaussian distribution with small variance centred on an intensity value is used to model the image intensities corresponding to each tissue type. Ideally, this model should suffice to establish a classification of the voxels into tissue types, but real MR acquisitions pose two problems, namely corrupting bias fields, which modify the intensity of a same tissue voxel depending on its location, and partial volume effects due to the presence of more than one tissue type in a single voxel.

The intensities present in a MR image are often corrupted by a multiplicative, spatially varying bias field $[9,11]$. Many techniques have been proposed to deal with this problem $[2,8,19]$, and the one we use is described in [11], being a modification of the method introduced by Wells and Grimson [20]. The technique uses the E-M algorithm to estimate iteratively the bias field and, for each of the voxels in the image, the probability that it belongs to each tissue type. Tissue types are modelled as Gaussian distributions, and a zero-mean Gaussian model is also assumed for the bias field. Figures $1 \mathrm{~A}$ and $1 \mathrm{~B}$ show a slice of a MRI and its corresponding corrected image. The estimated bias field is shown in figure $1 \mathrm{C}$.

An image segmentation into tissue classes can be obtained by assigning to each voxel the tissue type for which its probability is highest, that is, has maximum likelihood. However, in some cases, the maximum likelihood is still small, 
typically because the intensity value of the voxel after bias correction does not fit well to any of the tissue distributions. These voxels are regarded as containing several tissues, that is, exhibit the partial volume effect (PVE) [16]. Intuitively, voxels belonging to the boundaries between tissues are likely to be most affected. Indeed, we can consider low maximum likelihood voxels as good candidates for boundary voxels. Figure 1D shows the segmentation using explicit Gaussian tissue models for grey matter and white matter, together with a uniform extra class to cater for background and CSF [11]. PVE voxels are coloured white.
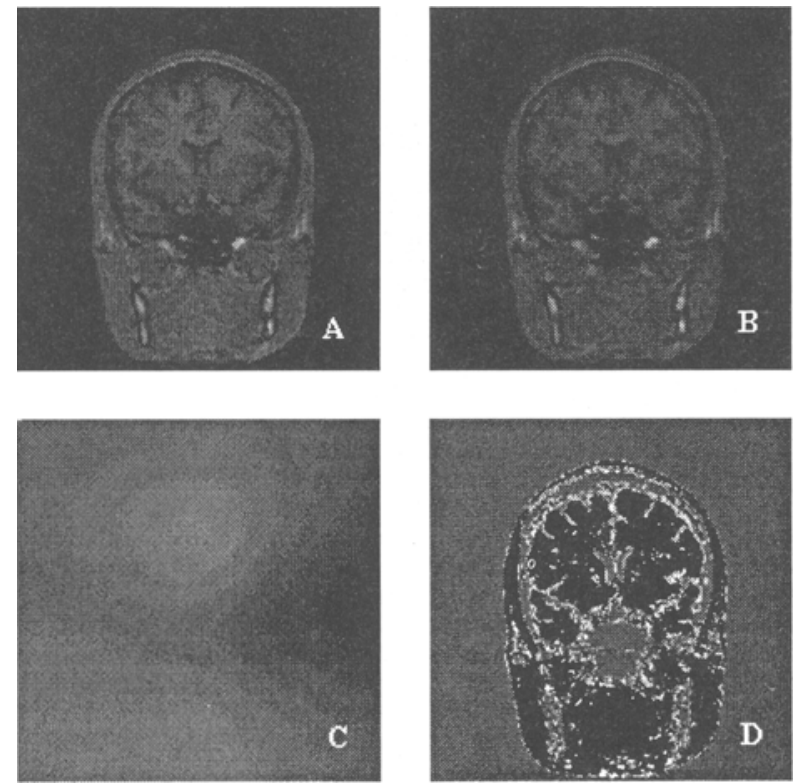

Fig. 1. A) Original slice; B) corrected slice; C) estimated bias field; D) segmentation using a tissue model for white matter and grey matter, plus a uniform class for CSF, air, and other tissues (PVE voxels are coloured white).

\section{Geometrical 3D segmentation}

Geometrical segmentation aims to detect the outer surface of the structure to be segmented. The advantage of such techniques is that they enable the specification of prior knowledge about the expected topology and shape of the structure by including an initial average model and a description of the allowed variability in shape $[3,18]$. We use the simplex mesh [6] technique for 3D segmentation, then construct a continuous, smooth surface by means of triangular Gregory-Bézier patches [17]. Prior knowledge is included by applying a principal components analysis (PCA) on the nodes of the simplex mesh, as described later. 
A simplex mesh is a discrete shape model, formally defined as a set of 3connected nodes. A framework in which a simplex mesh is used to segment volumetric data is described in [6]. A set of internal forces model properties of the structure, such as its elastic behaviour or constraints on continuity of the normals of the nodes; similarly, external forces are implemented and make the model converge towards the target volumetric data. The mesh is initialised and subjected to these forces following Newtonian dynamics:

$$
m \frac{d^{2} P_{i}}{d t^{2}}=-\gamma \frac{d P_{i}}{d t}+F_{i n t}+F_{e x t},
$$

where $m$ is the mass unit of a vertex, $P_{i}$ is the position of the $i$ th node at time $t$, and $\gamma$ is a damping factor.

Several operations enhance the flexibility of the simplex mesh when segmenting three-dimensional data. Some can be applied to alter the topology of the mesh, so rendering it suitable for complex shapes or for scale-space approaches to segmentation. Additionally, the density of the mesh is updated by a refinement process which increments the number of nodes in areas where a high level of detail is required. A further attractive property of simplex meshes is that they are dual to triangulations.

Prior knowledge about the expected shape of the structure to be segmented can be introduced into the model by acting on the internal forces that drive the fitting process, but this is not a very flexible method. Marais [13] has developed a scheme in which a PCA is applied to the nodes of a set of meshes segmenting the same structure in different patients. The result of this process is an average mesh $\bar{M}$ and a set of eigenvectors containing the $n$ principal modes of variation, $\nu_{1}, \ldots, \nu_{n}$. Other meshes are then expressed in terms of these elements:

$$
M=\bar{M}+\sum_{i=1}^{n} \alpha_{i} \nu_{i}
$$

This process not only limits the amount of information required to describe an object (the set $\alpha_{1}, \ldots, \alpha_{n}$ of coefficients), but also restricts the allowed variability in shape, so avoiding unwanted excessive deviations from the average model. Although the simplex mesh has the advantages enumerated above, it is intrinsically not smooth, posing problems for volume estimation of smooth, highly convoluted surfaces, such as the cortex. To address this problem, a triangulation is built from the simplex mesh by adding a vertex in the centre of each face of the mesh and updating its position to the nearest data point in the direction of the normal of the face, by means of a local search. Then, smooth triangular Gregory-Bézier patches on each triangle are used to build a G1-continuous surface (continuity in the tangent plane) passing through the nodes of the simplex mesh, without the need to use interpolating polynomials of very high degree. 
Triangular Gregory-Bézier (tGB) patches [17] are tricubic surfaces in barycentric coordinates, defined by 15 control points (refer to Figure 2):

$$
\begin{aligned}
G B(u, v, w)= & u^{3} P_{0}+v^{3} P_{1}+w^{3} P_{2}+ \\
& 12 u^{2} v w P_{211}+12 u v^{2} w P_{121}+12 u v w^{2} P_{112}+ \\
& 3 u^{2} v(1-w) P_{01}+3 u v^{2}(1-w) P_{02}+3 v^{2}(1-u) w P_{11}+ \\
& 3(1-u) v w^{2} P_{12}+3 u(1-v) w^{2} P_{21}+3 u^{2}(1-v) w P_{22}
\end{aligned}
$$

where $0 \leq u, v, w \leq 1, u+v+w=1$ and:

$$
P_{211}=\frac{w P_{211}^{v}+v P_{211}^{w}}{w+v}, P_{121}=\frac{u P_{121}^{w}+w P_{121}^{u}}{u+w}, P_{112}=\frac{v P_{112}^{u}+u P_{112}^{v}}{v+u}
$$
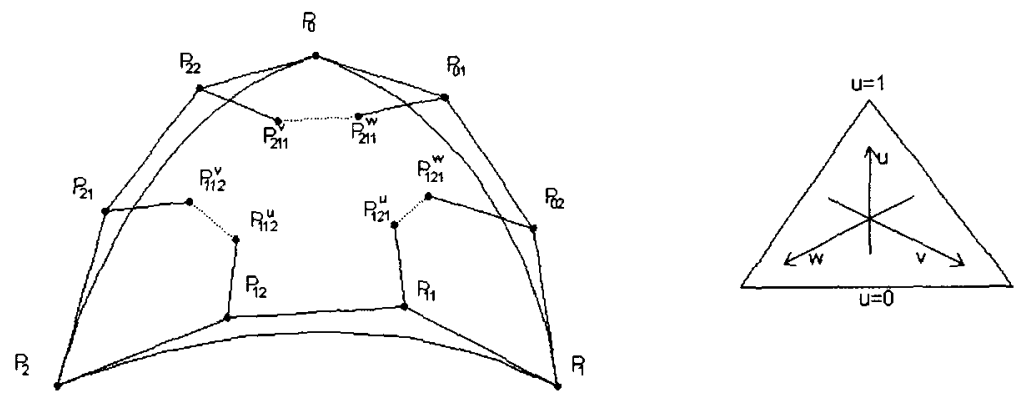

Fig. 2. Triangular Gregory-Bézier patch, defined by 15 control points. A G1-continuous mesh of tGB patches is used to interpolate the nodes of the simplex mesh and its corresponding normals.

The tGB interpolation process leaves one free control point in each of the edges of the triangulation, thereby enabling an even closer fit to the data. Several fitting strategies are being developed, namely local minimisation of distances from points inside a particular patch to the nearest data points, global minimisation of all the distances in the mesh, and a hybrid scheme where distances are minimised in a certain patch and its neighbouring patches up to a certain level, weighting the contribution of the neighbours as a function of the distance.

A desirable property of tGB patches is that analytic formulae for local position and derivatives are available, enabling the computation of shape descriptors based on differential geometric quantities, such as curvature. In a similar vein, the surface area and volume enclosed by a simplex mesh can be computed analytically using the divergence theorem. The availability of these measures is important in the development of quantitative shape descriptors applicable to medical structures, which is one of the most important ongoing lines of research in medical imaging. 


\section{Edge search and confidence bounds}

We assume that PVE voxels correspond to boundary voxels. This information initiates the simplex mesh, which looks for the closest boundary voxel when updating its nodes. This search samples the neighbouring voxels in the direction of the normal of the node, by means of fast, local, 1-D searches, similar to those used in [1]. The same mechanism is used when computing distances from patch points to data points in the process of fitting tGB patches to the simplex mesh.

Due to the partial volume effect, boundary transitions from one tissue to another can occupy more than one voxel. The location of the boundary can thus be bounded by this volume, i.e. from pure voxels of one tissue to pure voxels of another tissue. This interval defines the confidence bound for the measurement. Two simplex meshes and tGB surfaces are computed, representing the upper and lower bounds on the position of the real surface of the structure being segmented. The upper simplex mesh estimate is built by creating a parallel surface tangent to the data points found searching from the centre of each polygon (Figure 3).
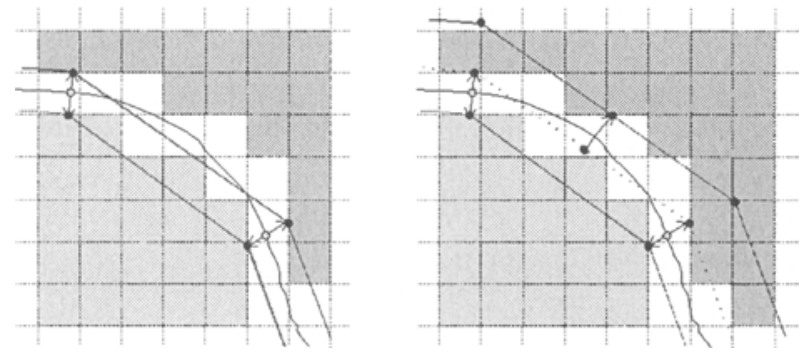

Fig. 3. 2D illustration of the 3D construction of the upper estimate on surface location using the simplex mesh. Upper and lower estimates on the location of the vertices of the mesh are obtained by local searches approximating to the nearest non-PVE voxel (left). Then, the position of the centre of the polygon (line in 2D) is updated to the nearest data point and a parallel surface is built (right).

\section{Results}

Several experiments have been performed in order to assess the performance of the method. Initially we used synthetic MRI data sets to validate the working of the program and to demonstrate that the tGB patches gave upper and lower bounds on volumes that are far tighter than those using voxel counting or the refined simplex mesh; [10] provides details and the imaging protocols that we simulated. Then we experimented with a phantom consisting of a group of shapes made from paraffin wax and embedded within an agarose gel. By measuring the density of the wax, the true volume can be derived from the weight within a 
confidence interval of $2 \%$ [16]. The phantoms simulate the size and shape of multiple sclerosis lesions, which are usually small relative to the MR acquisition resolution. Simplex mesh segmentation with low density, tGB fitting, and simplex mesh refinement volume measures were obtained. The results again show that the best volume bound estimates are from the use of a mesh of tGB patches interpolating the simplex mesh. It is worth noting that, although the refinement method of the simplex mesh improves the volume measurement significantly, the use of a continuous surface results in a much better estimate. These results are typical of the ones we have achieved with simulated and phantom data [10].

More recently, we have begun to test the method on an in-vivo T1-weighted MRI data set consisting of 124 slices of $256 \times 256$ voxels of size $0.781251 \times 0.78125 \times$ $1.7 \mathrm{~mm}^{3}(\mathrm{TE}=9000 \mathrm{~ms}, \mathrm{TR}=24000 \mathrm{~ms})$. In this case, no ground truth about the volume of the object to be segmented is available; for this reason we scanned the volunteer twice, the second time with his head rotated through about $20-30^{\circ}$ (Figure 4). Results are given for the left ventricle of the patient.
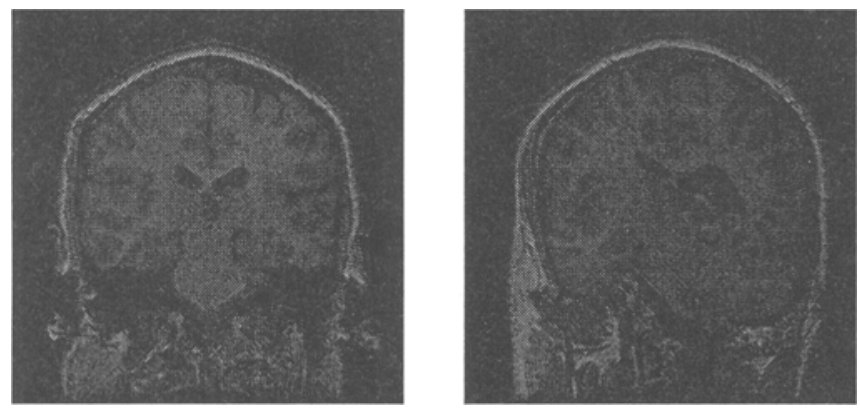

Fig. 4. Slice 56 of the MRI acquisitions used for the study: normal (left) and rotated(right). Note the significant rotation of the right image.

The data set is first bias corrected assuming only one tissue encompassing white matter and grey matter, plus a uniform class modelling the rest of tissues plus CSF and air. Probability maps for the different tissues are generated, and a pre-segmentation labels voxels with a probability smaller than $95 \%$ of belonging to one of the tissues as PVE voxels (see Figure 1). A simplex mesh is fitted to the data using the information of the map to guide it. Two meshes are fitted to obtain an upper and a lower bound ( $s m U$ and $s m L$ ) of the location of the surface. The numbers of vertices for the fitted refined mesh are 1538 (771 polygons), and 1558 for the rotated set (781 polygons). A set of tGB patches is then built $(t G B U, t G B L)$. For the sake of comparison, voxel-based segmentations were performed and validated by an expert using a thresholding tool and setting two thresholds in order to obtain upper and lower bounds (voxel $U$ and voxel $L$ ). Volume measurements are shown in Tables 1 and 2. 


\begin{tabular}{|l|c|c|}
\hline & straight & rotated \\
\hline smU & 12192 & 11100 \\
\hline voxel U & 10090 & 9770 \\
\hline tGB U & 9454 & 9574 \\
\hline tGB L & 7835 & 7998 \\
\hline sm L & 7373 & 7483 \\
\hline voxel L & 7314 & 7162 \\
\hline
\end{tabular}

Table 1: Measured vol. $\left(\mathrm{mm}^{3}\right)$ of left ventricle (initial and rotated).

\begin{tabular}{|l|c|c|}
\hline & straight & rotated \\
\hline sm & 4819 & 3617 \\
\hline voxel & 2776 & 2608 \\
\hline tGB & 1619 & 1576 \\
\hline
\end{tabular}

Table 2: Width of confidence interval (upper bound - lower bound).

Figure 5 shows the upper and lower bounds on the volume of the ventricle, and Figure 6 shows the width of the confidence interval. There are two points to note about Figure 6 . First, the confidence interval is significantly smaller for the tGB model, and second, the interval is almost invariant to the patient head rotation.

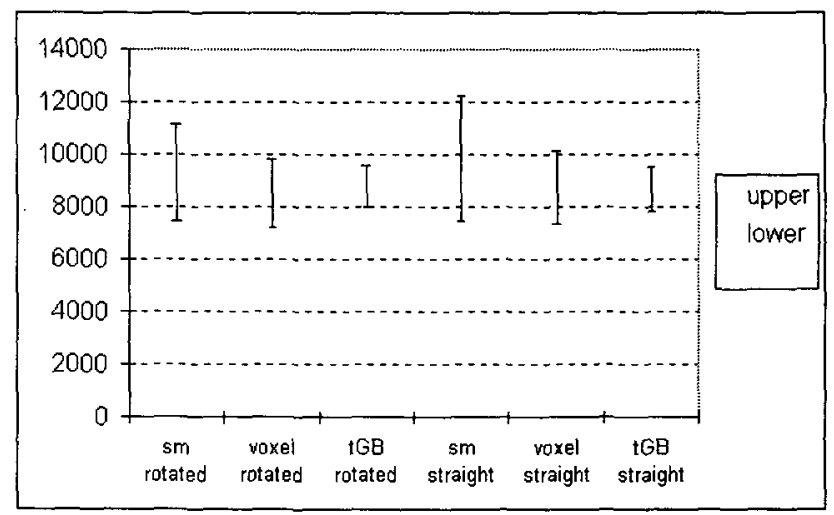

Fig. 5. Volume estimates for the left ventricle $\left(\mathrm{mm}^{3}\right)$, with the conditions indicated in the tables above, and for the original and rotated configurations. The vertical bars show the difference between the upper and lower volume estimates.

\section{Future work}

The method currently forms a framework for the segmentation and measurement of MRI data, leading to the estimation of two surfaces representing an upper and a lower bound on the location of the real surface. We are progressing towards estimating the most probable location of the surface inside the interval. PVE voxels can be modelled as weighted mixtures of two distributions on intensities. Using information about the neighbouring tissues, their distributions, and the intensity of the voxel, a probability distribution function (pdf) on the proportion 


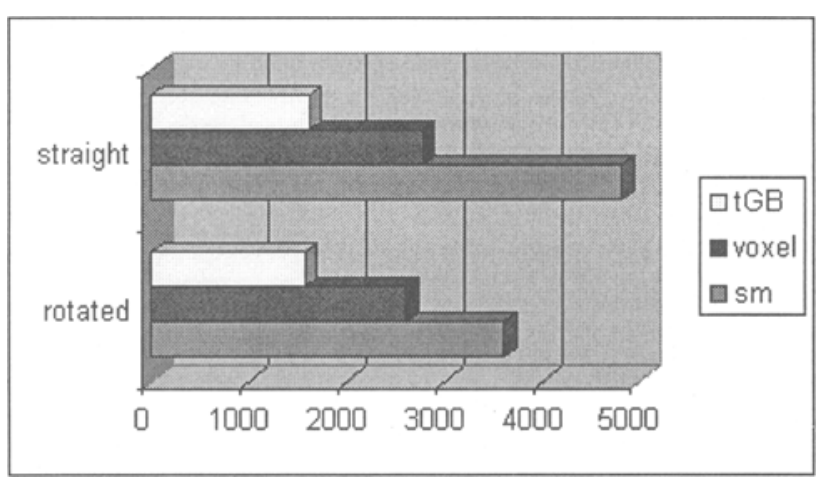

Fig. 6. Width of the confidence interval in the original and rotated condition. The best results are obtained by using a mesh of tGB patches interpolating the simplex mesh. Note that the results for the tGB patches are almost invariant to patient head rotation.

of each tissue present in the voxel can be computed. In turn, this will yield a pdf on the measurement so that both a mean/mode and a standard deviation can be computed.

The flexibility of the shape model is being investigated. A mesh consisting of a set of G1 continuous tGB patches leaves one degree of freedom on each edge connecting two patches. These free control points are currently set to minimise fluctuations on the surface, but a method that minimises the least-squares distance between the patch and target data points is being implemented. This scheme could be modified to minimise the difference between the proportions of each tissue in a voxel estimated as described above, and the proportions derived from the intersection of the tGB patches with each voxel.

Volume is by no means the most sensitive shape descriptor, and more sophisticated descriptors, suitable for medical applications, are being developed. The availability of a smooth continuous surface segmenting the object enables geometrical descriptors such as curvature to be computed and bounded. The segmentation method sketched in this paper shows an improvement over other existing techniques, in the sense that the confidence intervals are significantly narrower. An in-depth study is under way to assess whether this technique will be able to provide clinically valid measurements from MRI for problems such as segmentation of multiple sclerosis lesions or schizophrenia studies, where the size of the effect to be studied is small relative to the voxel size.

\section{Bibliography}

[1] Blake A., Curwen R., Zisserman A. A Framework for Spatiotemporal Control in the Tracking of Visual Contours. IJCV, 11(2):127-145, 1993. 
[2] Brechbühler CH., Gerig G, Szekely G. Compensation of Spatial Inhomogeneity in MRI Based on a Parametric Bias Estimate. Lecture Notes in Computer Science 1131, Proc. VBC'96, Eds: Karl H. Hoehne and Ron Kikinis, pp. 141-146, 1996.

[3] Cootes T.F., Hill A., Taylor C.J., Haslam J. The Use of Active Shape Models for Locating Structures in Medical Images. Image Vision and Computing, vol. 12, no. 6, jul. 94 .

[4] Colchester A.C.F., Gerig G., Crow T., Ayache N. and Vandermeulen D. Development and Validation of Techniques for Brain Morphometry (BIOMORPH). In Proc. CVRMED'97.

[5] Crow T.J. Temporal Lobe Asymmetries as the Key Etiology of Schizophrenia. Schizophrenia Bulletin 16, pp. 433-443, 1990.

[6] Delingette H. Simplex Meshes: a General Representation for 3D Shape Reconstruction. INRIA research report no. 2214. March 1994.

[7] DeLisi L.E., Tew W., Xie S., Hoff S.L., Sakuma M., Kushner M., Lee G., Shedlack K., Smith A.M., Grimson R. A Prospective Follow-up Study of Brain Morphology and Cognition in First-Episode Schizophrenic Patients: Preliminary Findings. Biol. Psychiatry 38, pp. 349-360, 1995.

[8] Gilles S., Brady M., Thirion J.P., Ayache N. Bias Field Correction and Segmentation of MR Images. Visualization in Biomedical Computing, Proceedings of VBC'96, vol. 1131 of Lecture Notes in Computer Science, pp. 153-158, Ed. Springer Verlag, 1996.

[9] González Ballester M.A. Computer-Aided MRI-Based Analysis of Brain Morphology. DPhil transfer report, University of Oxford, July 1997.

[10] González Ballester M.A., Zisserman A., Brady M. Combined Statistical and Geometrical 3D Segmentation and Measurement of Brain Structures. Proc. Workshop on Biomedical Image Analysis, Santa Barbara, Ca., 1998.

[11] Guillemaud R., Brady M. Estimating the Bias Field of MR Images. IEEE Transactions on Medical Imaging, vol. 16, no. 3, pg. 238, 1997.

[12] Kass M., Witkin A., Terzopoulus D. Snakes: Active Contour Models. Proceedings of $I C C V$, pp. 259-268, 1987.

[13] Marais $P$. In preparation.

[14] Marais P., Guillemaud R., Sakuma M., Zisserman A., Brady M. Visualising Cerebral Asymetry. Lecture Notes in Computer Science 1131, Proc. VBC'96, Eds: Karl Heinz Hoehne and Ron Kikinis, pp. 411-416, 1996.

[15] McInerney D., Terzopoulos T. A Finite Element Model for 3D Shape Reconstruction and Nonrigid Motion Tracking. In Proceedings of the Fourth Int. Conf. on Computer Vision (ICCV'93), pp. 518-523, 1993.

[16] Röll S.A., Colchester A.C.F., Summers P.E., Griffin L.D. Intensity-Based Object Extraction from 3D Medical Images Including a Correction for Partial Volume Errors. Proceedings of BMVC, pp. 195-204, 1994.

[17] Schmitt F., Chen X., Du W.H. Geometric Modelling From Range Image Data. Proceedings of Eurographics'91, pp. 317-328, Ed. Elsevier, 1991.

[18] Székely G., Kelemen A., Brechbühler C., Gerig G. Segmentation of 2-D and 3-D Objects from MRI Volume Data Using Constrained Elastic Deformations of Flexible Fourier Contour and Surface Models. Medical Image Analysis, vol. 1, no. 1, pp. 19-34. [19] Van Leemput K., Vandermeulen D., Suetens P. Automatic Segmentation of Brain Tissues and MR Bias Correction Using a Digital Brain Atlas. Technical report, Kathoelieke Universiteit Leuven, Leuven, Belgium, Jan. 1998.

[20] Wells III W.M., Grimson W.E.L., Kikinis R., Jolesz F.A. Adaptive Segmentation of MRI Data. IEEE Transactions on Medical Imaging, vol. 15, no. 4, pp. 429-442, 1996. 